\title{
Dichapetalum michelsonii Hauman. Nouvelle plante toxique pour le bétail du Ruanda-Burundi *
}

\author{
par D. THIENPONT ef M. VANDERVELDEN
}

La plante connue sous le nom vernaculaire de Umutambasha est une espèce récemment décrite par HAUMAN (1958) (1), mais dont les propriétés toxiques sont connues depuis longtemps par les autochtones comme une plante très dangereuse pour le bétail ef un poison violent pour l'homme. La décoction ou la macération des feuilles de cette plante serait mélangée à la bière indigène avec des intentions criminelles.

CURASSON (1942) (2) signale que la famille des Dichapetalacées comprend plusieurs espèces toxiques, qui, en Afrique occidentale, entrent dans la composition de poison pour flèches. II reprend les deux espèces toxiques décrites en Afrique du Sud (STEYN (1949) (3) : Dichapetolum cymosum (Hook) Engl et Dichapetalum venenatum Engl et Gilg.

Dans les territoires anglais voisins, on signale Dichapetalum brounii Engl et Krause qui est aussi réputé toxique. MARAIS cité par STEYN (3) a pu extraire le principe toxique de Dichapetalum cymosum. II s'agit d'un sel sodique ou potassique de l'acide monofluoro-acétique qui est extrêmement toxique et dont l'activité doit être apparentée à celle du groupe de la strychnine.

D'après les indigènes il existerait deux espèces d'Umutambasha : l'une étant toxique et l'autre pas. En réalité, il s'agit de deux plantes assez semblables dont l'une est Dichapetolum michelsonii ef l'autre, Uvaria angolensis, non toxique.

(*) Nous remercions M. VANDERBEN pour l'aide apportée à la rédaction de cette étude et pour la détermination des plantes.

Reçu pour publication : juin 1961.

Rev. Elev. Méd. vét. Pays trop. 1961, no 2.
Dichapetalum michelsonii (1) a une distribution géographique très étendue au RuandaBurundi. On la trouve aussi bien en altitude à plus de $2.000 \mathrm{~m}$ que sur les plateaux de $1.300 \mathrm{~m}$. Elle se présente sous la forme d'arbrisseaux groupés, à tiges droites et solides, avec des feuilles vertes, larges et luisantes.

\section{L'intoxication naturelle}

Les éleveurs rapportent des cas d'intoxication à n'importe quel moment de l'année mais spécialement pendant la saison sèche avec du bétail en transhumance. Selon eux, les vaches meurent brutalement après l'ingestion de quelques feuilles. Mais l'intoxication expérimentale du mouton prouve que la mort ne se produit pas instantanément. Selon d'autres, le bétail montre d'abord des signes nerveux ef meurt comme dans un accès d'apoplexie avec tétanie manifeste.

L'état du cadavre et des organes fait penser à une septicémie hémorragique aiguë.

L'évolution de la maladie serait plus rapide si le bétail avait bu après ingestion des fevilles toxiques.

\section{L'intoxication expérimentale}

1) du mouton por voie orale

$1^{\text {er }}$ cas : On administre $20 \mathrm{~g}$ de fevilles fraîches le premier jour, et $17 \mathrm{~g}$ le second jour.

Les premiers symptômes se déclarent brutalement 30 heures après le début de l'expérience : tremblements musculaires, grincement des dents, ataxie, opisthotonus, fétanie de tous les muscles, fibrillations cardiaques, le mouton tombe par terre, les pattes en extension rigide, le cou rejeté en arrière et il meurt brutalement. 


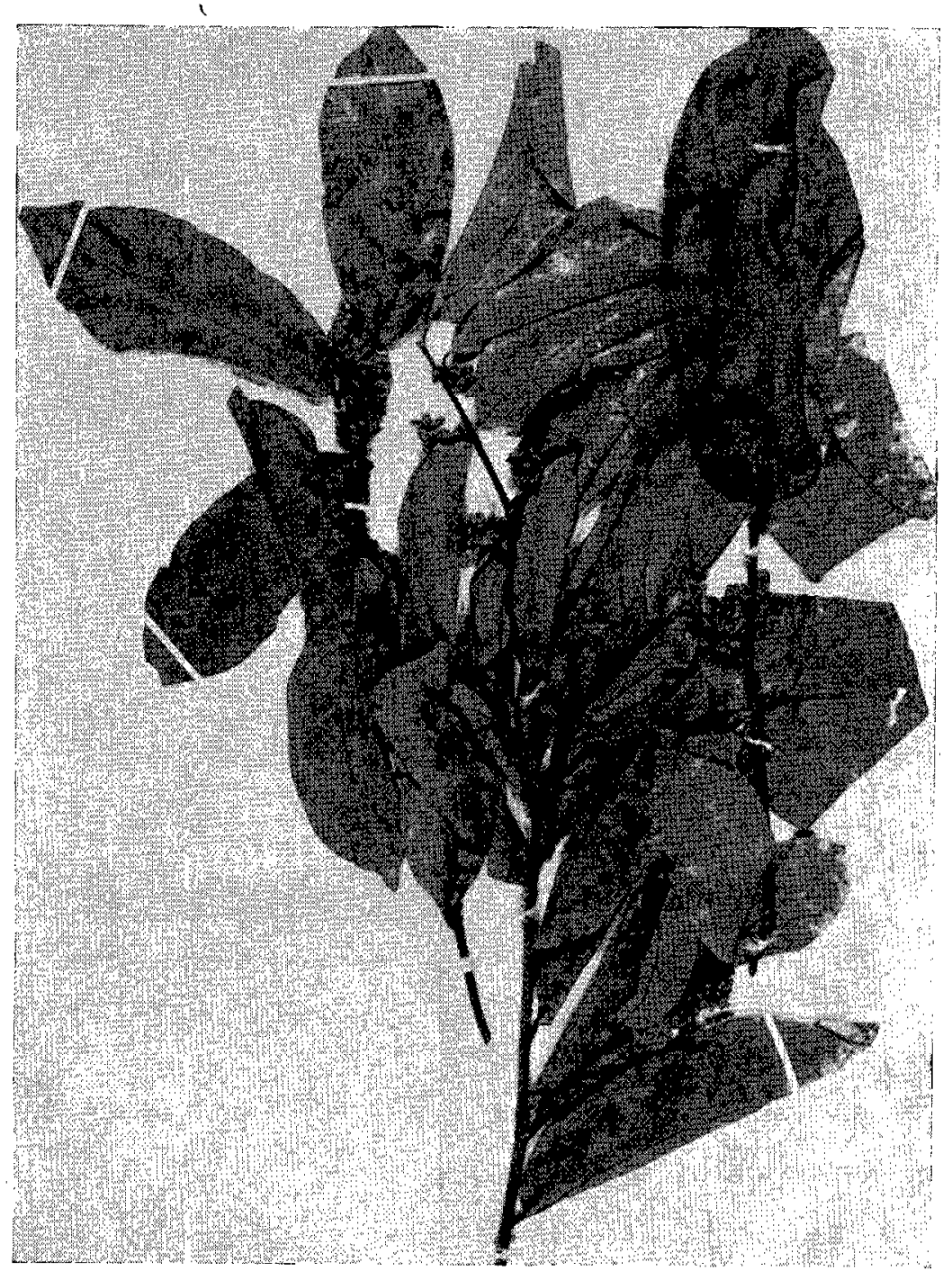

$2^{\mathrm{e}}$ cos : Ce mouton reçoit $20 \mathrm{~g}$ de feuilles toutes les 3 heures ; il meurt 48 heures après le début de l'expérience après avoir reçu $180 \mathrm{~g}$ de feuilles fraîches.

Les symptômes sont identiques à ceux du cas précédent.

\section{Lésions nécrotiques :}

Les lésions dans chaque appareil sont semblables et se caractérisent par la congestion, l'arborisation, les pétéchies ou les suffusions sanguines. Le cœur est spécialement atteint et montre des suffusions et des pétéchies sous l'endocarde et sous l'épicarde. Dans le tube digestif, les pétéchies sont le plus manifeste dans l'intestin grêle.
2) des cobayes

Le laboratoire de chimie de l'Institut tropical de Léopoldville a vérifié la toxicité de cette plante sur des cobayes. On a constaté la mort de la totalité des animaux en expérience par l'administration, chaque fois à deux heures d'intervalle, d'une dose de $5 \mathrm{ml}$ du filtrat d'une dćcoction aqueuse, titrée en poids volume à 10 p. 100 de poudre de feuilles.

La quantité de feuilles étant insuffisante, on n'a pas pu déterminer la composition du produit toxique.

Ecole des assistants vétérinaires d'Astrida Directeur : Dr D. Thienpont. 


\section{RÉSUMÉ}

Les auteurs signalent l'extrême toxicité de Dichapetalum michelsoni Hauman pour le bétail, le mouton ef le cobaye. Le principe toxique n'est pas connu, mais il agit sur le système nerveux central. Il provoque des symptômes qui font penser à une intoxication par la strychnine et des lésions du type hémorragique.

\section{SUMMARY}

\section{Dichapetalum michelsoni Hauman. A new toxic plant for the animals of Ruanda-Burundi}

The authors report the extreme toxicity of Dichopetolum michelsoni Hauman for cattle, sheep and the guinea pig. The toxic principle is unknown, but it acts on the central nervous system and induces symptoms not unlike those of strychnine poisoning.

\section{RESUMEN}

Dichapefalum michelsonii Hauman. Nueva planta tóxica para el ganado de Ruanda Urundi.

Los autores señalan la extremada toxicidad de Dichapetalum michelsonii Hauman para el ganado vacuno, la oveja y el cobayo. Nó se conoce el principio tóxico, sino que actúa sobre el sistema nervioso central. Provoca síntomas que hacen pensar en una intoxicación de estricnina y lesiones del tipo hemorrágico.

\section{BIBLIOGRAPHIE}

HAUMAN. - Bull. Jard. boton. Bruxelles, 1958, 28. I Vigot édit., Paris 1942.

CURASSON (G.). - Traité de pathologie exo- STEYN (D. G.). - Vergiftiging van mens en tique vétérinaire et comparée. Tome III. dier. Van Schaik édit., Pretoria 1949. 